\title{
Esthétique ordinaire et chats : ordinateur, corporéité et expression codifiée des affects
}

Anibal Frias

\section{(2) OpenEdition}

\section{Journals}

Édition électronique

URL : https://journals.openedition.org/tc/95

DOI : $10.4000 /$ tc. 95

ISSN : 1952-420X

Éditeur

Éditions de l'EHESS

\section{Édition imprimée}

Date de publication : 1 avril 2004

Pagination : 1-22

ISSN : 0248-6016

\section{Référence électronique}

Anibal Frias, «Esthétique ordinaire et chats : ordinateur, corporéité et expression codifiée des affects », Techniques \& Culture [En ligne], 42 | 2004, mis en ligne le 06 novembre 2007, consulté le 29 septembre 2022. URL : http://journals.openedition.org/tc/95 ; DOI : https://doi.org/10.4000/tc.95

Ce document a été généré automatiquement le 29 septembre 2022.

Tous droits réservés 


\title{
Esthétique ordinaire et chats : ordinateur, corporéité et expression codifiée des affects
}

\author{
Anibal Frias
}

\section{NOTE DE L'ÉDITEUR}

Je tiens à remercier Margarita Xanthakou pour son aide.

1 En moins de vingt ans, l'ordinateur est devenu en France un objet quotidien qui a envahi toutes les sphères de la vie sociale : professionnelle, administrative, domestique, artistique ou ludique. Il est porteur de potentialités technologiques, esthétiques et politiques insoupçonnées en matière d'économie, de communication, de création ou de contestation. Les contraintes techniques ou, plus positivement, le mode de fonctionnement que tout utilisateur du multimédia doit adopter n'ont pas limité la création langagière ni rendu uniforme le style scripturaire. Au contraire, l'absence des accents et des cédilles imposée par le modèle anglo-saxon, les abréviations introduites dans les écrits ont favorisé une certaine économie d'écriture sans entamer la compréhension du message, tout en encourageant la prolifération des «lieux» d'expression, des paroles, des codes, des symboles, des dessins, tout cela supposant un apprentissage des usages et une coopération réglée.

Du même coup, tout en étant individuellement approprié, l'outil informatique suscite des modalités du lien social virtuel, à travers une téléprésence qui relève des échanges, des sociabilités, du divertissement ou de l'érotique et dont n'est pas absente une inventivité subjective et collective. En même temps, il fait osciller le jeu du réel et de l'irréel, de la cognition et de l'action, du sens et des sens.

3 Il est convenu de voir dans les chats, ces espaces de discussion sur Internet aux contours ludiques et informels, un mode de communication médiatisé par l'ordinateur. Or, bien plus qu'une relation de communication et qu'un medium technique, on a affaire 
à un univers culturel et créatif cohérent où l'ordinateur est coextensif aux usages : il informe les gestes et les opérations cognitives comme, de son côté, la sensorialité évolue intimement avec l'artefact domestiqué, voire humanisé.

4 Je me propose d'aborder dans cet article ${ }^{1}$ l'investissement des chats. Il s'agit plus précisément de rendre compte de la dimension créative "spontanée » et interactive, dans un "cadre technologique » socialisé, et même codifié par la Netiquette, l'éthique en vigueur sur Internet. Tout en reconsidérant dans un premier temps la notion d'esthétique ordinaire, l'étude porte sur les usages et les significations des smileys, ces graphismes conventionnels qui expriment les sentiments et les affects des internautes au cours des échanges. Contrairement à l'idée d'une disparition (post)moderne du corps et d'un dualisme cartésien âme/corps soutenue par David Le Breton (1999), je montrerai qu'avec l'avènement du numérique, la corporéité (trans)figurée reste un vecteur symbolique central dans les échanges scripturaires sur les chats comme dans les usages cognitifs de l'ordinateur.

Une esthétique matérielle : le monde sensible

5 Que serait un monde réduit à sa pure phénoménalité, à son intelligibilité ou à sa virtualité ? On peut l'imaginer ressemblant à l'environnement de l'animal, à l'univers d'un dieu géomètre ou à la réalité de l'ordinateur. Le monde socialisé suppose que l'homme l'habite par le savoir et le sens mais aussi par l'activité et les sens. Si bien que pour nous, le monde est une configuration sensible. Celle-ci peut être définie en reconsidérant la notion d'esthétique.

6 La modernité a séparé l'art des autres domaines de la vie. En l'enfermant dans les temples muséaux et les galeries, en le réservant aux artistes et aux connaisseurs, elle en a aussi déterminé la logique de production, de circulation et de présentation, comme elle a pu jouer sur les conditions d'accès à son égard et les manières de ressentir.

7 Or depuis quelques années s'est opérée une déterritorialisation de l'art. En annexant la sphère publique et même les espaces interstitiels comme la rue, le terrain vague, l'usine ou l'abattoir désaffectés, jusqu'au domicile et l'hôpital; en colonisant des surfaces quelconques, comme le mur ou le trottoir, les œuvres relèvent de modalités expressives singulières, accèdent à d'autres publics et sont le fait de créateurs renouvelés. Au XXe siècle, le travail ou l'exposition de "sous-objets" ouvrent d'autres perspectives à l'artiste : matériaux bruts, urinoir, papiers, déchets matériels ou corporels. Les médias et le multimédia ont à leur tour été intégrés à ce champ créatif en tant qu'outil, dispositif, support ou objet d'art² ${ }^{2}$. Depuis Kant, l'esthétique est associée -et réduite- à l'art, en relevant du jugement de goût et d'un pur jeu des formes. Sont évacués comme «barbares » les éléments émotionnels et matériels, autrement dit les sensations et le sensible (Kant 1982: § 13). Une telle association est aujourd'hui problématique: des artistes récusent la notion d'esthétique, des projets en questionnent la pertinence. Et du coup, sont imaginées des créations inédites qui laissent entrevoir, en creux, des expériences sociales « autres». Peut-on d'ailleurs concevoir un art qui ne s'enracine pas dans l'existence et qui ne serait ni motivé ni orienté (ce que Kant appelle l'« attrait ») par quelque désir, valeur ou affect?

8 Mais c'est l'esthétique même qui peut être envisagée séparément et différemment. En effet, elle semble obéir à une logique propre et s'étendre à toutes sortes d'espaces et d'activités. En dehors d'une "intention» artistique, cette esthétique relève de l'invention des pratiques, de l'expérience sensible, d'un jeu avec les éléments ou les mots. Elle tient moins de la contemplation solitaire que du vécu et de la relation à 
l'autre, pointant une esthétique de l'existence et des formes de vie. Loin d'être pure et désintéressée, elle engage les sujets dans un être-ensemble socio-affectif, irréductible à la fonctionnalité et à la rationalité. Cette esthétique sociale sourd du quotidien. Ce qui relève de l' " attrait ", de la sensation comme du sensible, exclu par l'idéalisme kantien comme par tout formalisme, se trouve en fait inclus dans les relations entre les sujets et les objets, ou entre les sujets eux-mêmes.

9 Partant, l'esthétique est irréductible à l'art. Elle est plus et autre chose que de la création : elle est créativité et expressivité. Elle pointe un sens commun débordant le jugement esthétique, auquel Kant l'a réduite ${ }^{3}$, pour constituer une trame signifiante qui fait tenir ensemble une communauté de personnes. Elle est une dimension essentielle tant de la société que de la subjectivité. Art de vivre, elle transcende les instances matérielle et fonctionnelle pour faire affleurer un mode relationnel où se mêlent sensibilités et valeurs. Par-delà une pratique limitée: la création, et une sphère particulière : l'art, l'esthétique tient du vécu et s'avère une "forme " constituante de l'existence. En insistant sur la dimension relationnelle de l'art contemporain et notamment les installations matérielles ou numériques d'artistes, Nicolas Bourriaud montre, contre certains jugements hâtifs, qu'il s'agit d'un art incluant, dans le concept d'œuvre, le public, lequel passe de spectateur à acteur ; ce faisant, on a affaire à un art "inter-humain" qui vient questionner, par une "esthétique relationnelle», l'uniformisation sociale et la réification économique des relations courantes. Le passage -esthétique, social et épistémique- d'un art confiné à la « représentation » à celui de la "participation» active laisse entrevoir de nouveaux liens en inventant, dans l'interstice des «zones de communication» aménagées, des formes sociales autres de l'être ensemble. C'est pourquoi selon l'auteur, un tel art, loin d'être "nul », est au contraire "politique» dans sa démarche, sinon son intention, grâce à l'expérimentation in concreto d'un imaginaire de vie collective (Bourriaud 1998: 17 et n. 11-41).

10 Jean Caune (1997: 4) soutient que "le phénomène esthétique ne se manifeste plus seulement dans l'ordre de l'art, il concerne de multiples domaines de l'activité et de l'expérience humaines ». L'auteur l'étend au vaste champ de la communication, celle-ci apparaissant entrevue sous l'angle élargi de l'esthétique puisqu'elle ressortit aux "phénomènes de relation sociale du point de vue du contact et du lien sensibles " (Caune 1997 : 6). Cette esthétique de la communication se joue à l'interface expression/ expressivité en impliquant les éléments relationnels et dramaturgiques, tels que langage, gestualité ou art. De son côté, Abraham Moles (1990:17) a pu parler d'une "esthétique informationnelle» à l'égard de tout type d'expression artistique, et notamment du composé art-ordinateur. L'auteur conçoit l'œuvre comme « un message prélevé dans un ensemble socio-culturel et transmis par l'intermédiaire d'un canal (système de sensations visuelles, auditives, etc.) entre un individu -ou un microgroupe créateur-, l'artiste émetteur et un individu récepteur ». Son étude porte néanmoins sur le seul registre de la communication auquel l'art est assimilé, à savoir le «contenant» technologique («l'emballage», les signaux, le codage) aux dépens du « contenu » (le sens, l'émotion) qui est évacué.

11 En fait, c'est l'existence de tous et de chacun qui est traversée par une beauté «triviale» et accessible : la couleur de la tasse du petit déjeuner, la courbe d'un meuble, le matériau d'une maison, une ritournelle, la confection d'un paquet, le style d'un habit, le revêtement d'un cahier ou d'un mur, la rhétorique d'une conversation, la 
réalisation d'un bouquet, une gestuelle, la manière de servir le thé ou bien les usages créatifs et ludiques de l'ordinateur. Cette esthétique du quotidien se rapporte sans doute au « bricolage » constitutif d'un « art de faire » (de Certeau 1990). Elle avoisine en outre les formes sensibles de la vie sociale, saisies par le biais de l'imaginaire collectif. Ces formes sensibles sont "rendues» par l'observateur selon une méthode " poétique ", attentive à retenir dans le discours cela même qui échappe au rationnel: le substrat qualitatif que sont couleurs, odeurs, saveurs, sonorités et autres sensations tactiles (Sansot 1986 et 1995). Autant de strates du réel ou de la mémoire qui, conjuguées aux images, retiennent par-devers elles un fragment, une trace du monde phénoménal. Par elles, la société nous apparaît comme un composé d'actions et de sensations, seulement isolables in abstracto, et moins comme un ensemble d'entités. Une telle morphologie sensible pointe une épaisseur, une amplitude et une rythmicité d'où se dégage un corps social vivant, dynamique et tout en volume.

Cyberliens et technoculture

12 Ce qui précède suggère que la créativité sociale est irréductible à la création artistique tout comme l'expressivité sensorielle ne saurait se limiter à l'expression communicante $^{4}$. De la même manière, l'interactivité sur ordinateur reposant sur ce que l'on pourrait nommer des "techno-liens ", appelle une interaction par quoi surgissent les aspects sociaux et symboliques. Cet aspect a été souligné par Anne Sauvageot (1996: 216) :

«Au concept d'interactivité (l'homme-machine) se superpose celui d'interaction qui implique toute la gamme symbolique des échanges, par machines interposées, des hommes entre eux ».

13 L'interactivité dans le domaine du multimédia n'a de signification sociologique que sous l'angle d'une mise en culture du technologique. Loin de se réduire à un opérationnalisme machinique, auquel renvoie le terme numérique, il favorise au contraire des mises en relation, des interconnexions, des gestes créateurs et signifiants. Et finalement, un nouveau monde social et culturel, la cyberculture ${ }^{5}$, non pas « froid » à l'image de la technologie, mais en partie sensible et humain.

14 Ce type d'interaction reflète une " technoculture " porteuse d'un « techno-imaginaire » (Berger 1996: 148). Il intervient sur un mode transitif en "passant par » un registre culturel, esthétique ou matériel avec le rôle des objets ${ }^{6}$. L'ordinateur devient à son tour un objet relationnel. Le numérique intervient, sur le registre virtuel, comme mode d'appropriation du monde, à côté des modes religieux, économique, langagier, intellectuel, artistique ou pratique (Marx). Peut-être concentre-t-il tout cela à la fois ? Le multimédia ressemble à ce que Pierre Lévy (1998: 74) appelle, à propos de l'objet technique, un opérateur de virtualisation. Le monde auquel chacun a formellement accès est désigné comme cyber-espace, réalité virtuelle ou plutôt « virtualisation $»^{7}$. Le numérique suscite une esthétique relationnelle, un techno-art ou des expérimentations se déployant selon une ligne de déterritorialisation qui déborde et contourne l'institutionnel et le rationnel pour aménager des espaces interstitiels et nomades.

Si bien que cette interactivité doit être considérée comme une nouvelle « forme " sociohistorique du lien ouvrant sur des potentialités sociales, cognitives, artistiques ou émotionnelles et aussi sur des configurations subjectives et collectives singulières. Par là, elle crée de nouveaux objets et libère des expériences de vie: savoirs, êtres, communications, droits, pouvoirs, marchés, créations, identités, corps, jeux, érotismes, (in)dépendances. Ce qui se déplace, ce sont les catégories et les frontières du sujet et de 
la communauté, du réel et de l'imaginaire, du local et du global... Il faut toutefois distinguer l'idéologie moderniste ou technophile, concevant la réalité sociale ou historique en termes dualistes et discontinuistes (changement/reproduction, rupture/ continuité, innovation/archaïsme) et le relatif enchaînement des choses, la consécution des pratiques qui s'inscrivent, plus qu'il n'y paraît (à en croire nos représentations) dans une trame expérientielle pétrie d'habitudes de pensée et de routines d'action sans pour autant céder à l'illusion reproductiviste.

Cela dit, nous pouvons nous demander si l'on n'a pas affaire à une configuration sociotechnique moderne incarnée par le numérique qui, peut-être, va -est en train de - redéfinir ce qu'est l'Homme. En quoi ce composé débouche-t-il sur des agencements inconnus, sur des pratiques inattendues et sur une figure anthropologique " autre»? C'est ce que, dans le prolongement de cette ligne interrogative, Pierre Lévy (1998: chap. V) appelle une "poursuite de l'hominisation ». Ce devenir humain de l'homme relève d'un registre social, symbolique et technique évolutif en ce sens que surgissent toujours de nouvelles combinatoires idéelles, des connections logiques, des assemblages matériels, des potentialités et des forces qui font émerger un "donné » inédit, extériorisé et intériorisé selon une dialectique de l'objectivation et de la subjectivation.

Bien que Pierre Lévy (1998 : 73) critique la conception naïve d'un outil prolongeant le corps, on peut reprendre ici l'idée d'André Leroi-Gourhan (1965 : 88) lorsqu'il affirme :

«L'homme fabrique des outils concrets et des symboles. Les uns et les autres recourent dans le cerveau au même équipement fondamental [...]. Le langage et l'outil sont l'expression de la propriété de l'homme ".

Un tel processus historique et socio-cognitif procède par cannibalisation et domestication de la technique. La logique de cannibalisation s'effectue par " couplage $\|^{8} \mathrm{du}$ corps et de la machine donnant lieu à un composé unitaire; elle s'accomplit par subjectivation (collective), c'est-à-dire par incorporation d'une entité extérieure aux habitudes sociotechniques et mentales d'une société, tout en s'ajustant au «style» ou à l'«habitus» collectif, au sens de Leroi-Gourhan. Ce mouvement d'hybridation et de greffe a pour effet que la "machine n'apparaît plus comme machine » (Berger 1996 : 149). La logique de domestication s'indexe sur la première. La domestication concerne la dynamique d'appropriation qui fait passer un artefact de la société (ou du marché) à l'univers du familier par quoi un objet, écriture, téléphone, voiture ou ordinateur, devient un besoin "nécessaire » dans l'économie quotidienne, transformant en retour les gestes, la pensée, les affects, les relations. Par captation subjective et collective, l'artefact finit par être vécu sur un mode naturel, en devenant une " seconde nature ». Entre l'homme et l'environnement technique s'établit alors une sorte de « connaturalité » selon Gilbert Simondon (1989: 89), puisque ce lien est intégré à l'expérience, étant transformé, par cette même alchimie culturelle, en « nature ».

On le voit, l'ordinateur, comme du reste toute technique, est plus et autre chose qu'un simple medium, support ou instrument. Il remodèle l'humaine nature, le sens du vécu et le jeu du savoir et du pouvoir 9 . Son usage social suppose de recourir au corps dans ses dimensions expressives.

Corps sensible et ordinateur

20 En tant que configuration du corps, les perceptions sensorielles entremêlent affects, sens, action et cognition. Les sens de l'individu retiennent des informations concernant l'environnement physique et social et permettent d'agir ou de réagir aux situations 
labiles de la vie courante (Simmel 1981 ; Ninio 1989 ; Classen 1997). Ils participent de la socialisation et de l'adaptation. Leur fonction appréhensive est de nature centripète. La sensorialité a aussi une face expressive et préhensive, voire motrice. Elle est d'ordre centrifuge. Avec l'apparition de l'ordinateur et des communautés virtuelles, certains ont pu annoncer la dissolution du « réel », la dissémination du local et la disparition du corps au profit du seul registre visuel. Qu'en est-il exactement?

21 Le numérique concerne les «immatériaux » que sont images et textes virtuels. Leur réalisation suppose de passer par le digital, c'est-à-dire par la matérialité et la sensation du toucher : les doigts de la main et la tactilité. Cette matérialité et cette sensorialité intègrent un schéma cognitif. Ce fait peut sembler évident et de bon sens. En le rappelant, il a le mérite de signaler qu'entre l'ordinateur et le sujet, autrement dit entre les algorithmes de la machine et l'intellection humaine s'intercale un tiers relais. Celui-ci relève de l'opérationnalité technique et idéo-gestuelle comme de l'appréhension sensitive et de la symbolisation. Si bien que l'interactivité met en présence non pas deux mais trois éléments: technique, intellectuel et sensitif; l'artefact, le conceptuel, le corporel ${ }^{10}$. L'écran devenu tactile (visuel et sonore) peut à lui seul cristalliser ces registres. À la jonction du concept et de l'artefact, le corps sensible est ici substrat et medium.

Le tactile, le visuel, l'écoute, la voix quelquefois et bientôt l'olfactif ${ }^{11}$ sont autant de percepts et de sources d'affects qui composent avec le fonctionnement socialisé de la machine. L'intellection visualisante recourt à la représentation matérielle et à l'inscription graphique, comme par exemple le besoin d'imprimer un document pour percevoir autrement et le toucher physiquement (cf. l'expression " retoucher un texte " liant le contact manuel et le travail intellectuel) ${ }^{12}$.

De même, le langage de la manipulation matérielle n'est pas absent dans l'interaction avec l'ordinateur. L'abstraction des opérations appelle leur «manuelisation" symbolique et verbale : l'expression "avoir la main » signifie que, par le biais de la souris, l'opérateur retrouve l'usage du curseur avec la réapparition de la «flèche » sur l'écran, après que l'effectuation d'une procédure l'a rendu momentanément indisponible. Certains vieux MacIntosh d'ailleurs utilisent toujours une "vraie » main stylisée: les doigts ouverts désignent la préhension possible lorsque l'accès aux fonctions est à nouveau permis; fermés, il faut patienter jusqu'au terme de la procédure automatique; les doigts figurés servent encore à décompter le nombre de secondes qu'il reste pour, justement, «avoir la main » - une expression de maitrise et de puissance qui est finalement restée en devenant métaphore d'un langage technique, ayant survécu à la disparition du référent « réel »/virtuel, remplacé actuellement par un simple curseur. Ce processus socio-technique montre que plus un objet devient familier, plus ses fonctions socio-graphiques codées et représentées sont abstraites.

L'illusion est ici continuiste (ordinateur-cerveau) et simultanéiste ("temps réel»), provenant, d'un côté, de la vitesse avec laquelle l'œil capte une opération de la machine et, de l'autre, du fait que, intégré à nos habitudes, il n'oppose guère de résistance à nos gestes et désirs. Aussi ne pense-t-on pas «avec » ou «au moyen » d'un ordinateur, du moins lorsqu'il nous est devenu intime ${ }^{13}$, voire consubstantiel, mais dans sa logique de fonctionnement et dans son temps d'action en "s'installant» en lui, comme dirait Merleau-Ponty. C'est pourquoi l'ordinateur est un type d'" artefact cognitif ", au sens de Donald Norman: "un outil artificiel conçu pour conserver, exposer et traiter l'information dans le but de satisfaire une fonction représentationnelle » et qui assure 
souvent «des opérations et des représentations qui ne seraient pas faisables dans le monde réel ». Un tel dispositif artificiel, s'il amplifie les capacités intellectuelles -et physiques- humaines, change en même temps la nature de la tâche exécutée (Norman $1993: 18$ et 21$)$.

Le lien entre le «dedans" de l'ordinateur et l'environnement extérieur a progressivement évolué et favorisé des interactions. Séparés en un premier temps, ils évoluent pour constituer une interface. C'est ce que note Edouard Couchot (1996:128) :

«Les écrans ont permis d'abord de voir ce qui se passait à l'intérieur, dans le déroulement des programmes "; tandis que des dispositifs toujours plus nombreux «crayons électroniques, souris, manches à balais, boules roulantes, gants et vêtements de données, capteurs de plus en plus divers permettaient d'y introduire des informations autrement que par le clavier.»

Ces éléments garantissent une manipulation des fonctions de la machine, par toute une gestuelle, et permettent d'éprouver des sensations. La maîtrise passe ici par une préhension sensitive autant qu'intellectuelle. La porosité de ces interfaces fait s'interpénétrer la chair du sujet et le corps de l'objet en une inextricable hybridation. Certaines innovations informatiques prennent davantage en considération les moindres nuances de l'univers humain, en particulier les mouvements du corps qui peuvent être reproduits par l'outil numérique et modifiés, sur l'image, comme autant de possibles réalités imaginaires, de modes d'être et de mondes. Ainsi, en pénétrant plus avant dans le virtuel et l'abstraction, ces découvertes permettent d'embrayer sur l'« extérieur » et d'agir sur lui à travers l'ordinateur, médiatisé par la corporéité. S'il y a, "d'une certaine manière, dématérialisation dans les rapports de l'homme à la machine et au réel, on voit naître aussi, à l'inverse, la possibilité d'introduire au sein des algorithmes [...] des informations directement issues du réel, et du corps en particulier (gestes des doigts, des bras, des jambes, du visage, mouvements et déplacements dans l'espace, actions diverses, etc.) » ou "des informations (auditives, haptiques, proprioceptives)» (Couchot 1996 : 128). Ces informations mettent en jeu une large partie du sensorium du sujet. La technologie intègre également la part subjective, indissociable d'une logique floue positive et d'un apprentissage concret : "Les logiciels ne proposent pas seulement à l'utilisateur des repères conventionnels: signets, raccourcis, paramétrages [...], ils présentent aussi des plis, des configurations fortement singulières et contingentes dépendant de l'ordinateur, de son utilisation et de l'environnement internet » (Le Long 2002 : 286-287).

Dans le cas du numérique, l'écran de l'ordinateur ou le plan d'un dispositif sont disponibles comme surface de création et interface de rencontre. Une machine qui intègre la variable 3D, prenant en compte l'écrit et la voix, les émotions et expressions subjectives d'une façon synesthésique, tend à devenir « réaliste » en se complexifiant et, sans paradoxe, en se "déréalisant ». Mais « en retour, à travers ses interfaces, la machine nous remodèle un autre corps [...]. Le corps altéré que nous renvoie la machine s'organise autour des nouvelles perceptions, des nouvelles synesthésies, qu'elle provoque» (Cauchot $1996: 131$ ).

Expressivité et affects : les smileysLes notions de chat et de smiley

On peut définir le chat comme un «cadre technologique " (Sauvageot $2003: 270$ ), en ce sens que les cadres sont « coconstitutifs de nos conditions d'existence. En les étendant aux artefacts de nos modes de communication et de connaissance, ils définissent aujourd'hui les conditions du possible et du vraisemblable, les conditions même de 
l'artifice. Les cadres sont ni plus ni moins nos environnements techniques disposant, pour nous, en nous et avec nous, de la réalité de nos actions [...]». En outre, pour l'auteur, les cadres relèvent à la fois du technique, du symbolique et de l'institutionnel :

«Les cadres rapportés à des environnements techniques impliquent tout à la fois un potentiel technique -autrement dit, les conditions effectives d'interactions-, un potentiel symbolique -autrement dit, le positionnement du sujet dans la "réalité" de son expérience, ses valeurs et ses normes-, un potentiel institutionnel -autrement dit, les règles déontologiques et juridiques d'actualisation. »

Justement, les smileys relèvent de ce " potentiel institutionnel ». Ils sont apparus avec Internet, en particulier dans les forums de discussions conviviales à plusieurs (chat: bavarder -en «temps réel » sur le réseau). C'est de la nécessité d'une réglementation (la Netiquette) des sites de rencontre, provenant des Internautes eux-mêmes, qu'ils sont apparus peu à peu et se sont imposés. A priori, ils semblent moins présents sur le courriel. Peut-être cela est-il dû, d'une part, à la relative interconnaissance entre " emailiens", laquelle ne se réduit pas à une interactivité entre membres d'une « communauté virtuelle " (Rheingold 1995) où la communication, quoique désincarnée, repose parfois sur une interaction "réelle "; et d'autre part, à une connexion plus fonctionnelle, bien que la part ludique y soit présente. Ces raisons ont pour effet de diminuer le risque d'un langage ambigu et, partant, de limiter les conventions linguistiques ${ }^{14}$. Il reste que les smileys tiennent d'un art de la discussion relevant d'un style oral, d'un jeu avec la langue ou avec le sens des mots. Ils participent d'une recorporéisation des échanges et d'une esthétique "minimale ». Autant de manières subjectives, colorées de dire ses pensées et ses émotions et d'usages collectifs tenant de l'expressivité.

30 Le terme générique smileys dérive du verbe anglais to smile: sourire. Forgés au départ avec des signes de ponctuation et des caractères d'imprimerie, ces pictogrammes forment maintenant, dans leur usage, un code typographique. En intégrant l'écriture internet, ils relèvent d'un sociolecte fait de codes, d'acronymes, d'abréviations qui procèdent, en fait, d'un langage cryptographique mis au point par les services secrets d'un pays européen durant la Guerre Froide (Benedito 2002: 125). Ces symboles conventionnels sont appelés "emoticons", contraction de «emotional» et «icon». Les smileys «représentent de manière schématique (si on les incline à $90^{\circ}$ ) des mimiques faciales comme des sourires, des clins d'œil, des moues de colère ou de tristesse » (Marcoccia 2000 : 249). Conventionnels, stéréotypés et figurés, ils servent à rendre la "parole " plus expressive et vivante, à la manière des points d'exclamation ou de suspension. De tels icônes sont porteurs de renseignements sur les aspects relationnel et émotionnel de l'échange entamé par l'émetteur. À ce titre, ces codes relèvent du paraverbal puisqu'ils disent moins le sens que l'esprit dans lequel un message a été produit et comment il doit être interprété par le(s) destinataire(s). À la façon des «insultes rituelles» des jeunes Noirs des ghettos américains, étudiées par William Labov (1993), ces codes linguistiques et expressifs sont indissociables de la situation de communication, quoique ici l'interaction soit virtuelle et à distance. Les codes varient alors selon les interlocuteurs et le type de dialogue, comme ils supposent un public (même virtuel) appréciateur et évaluateur des performances scripto-verbales et des blagues ainsi que le partage d'un savoir commun. Ils fonctionnent, tour à tour, en tant que marqueurs de relation, adoucisseurs de menace (surtout face à des propos non univoques comme l'humour ou l'ironie), substituts à l'intonation, signes d'appartenance à la communauté des internautes et ses règles ${ }^{15}$. 
Types et formes de smileys

En fait, les sentiments, les émotions, l'affectivité, la proximité, la séduction, l'humour, l'ironie, la haine, le désenchantement ou la crainte sont des états d'âme saisissables à partir d'une épiphanie, c'est-à-dire un ensemble de signes. Ces états se reflètent dans un trait du visage ou une posture corporelle marqués, dans l'écriture virtuelle, par des figures composées de signes graphiques, des abréviations et des acronymes; ils sont en outre identifiables par certains mots chargés de signification et de sentiments (petits mots, diminutifs, surnoms, babillages, etc.), par des expressions (déclarations, formules, etc.), par des codes symboliques sexués accompagnés d'un dessin ou d'un message, par le recours à la taille des lettres, par l'usage de vocables étrangers (I love you, ciao, etc.), par la déformation ludique des mots, par l'emploi d'onomatopées suggestives, par l'alignement répété d'un signe de ponctuation ou d'une lettre (hé!!!!! ; ouaisssss). Parmi toutes ces techniques, retenons les smileys en tant que graphismes expressifs des affects. Notons les plus connus et employés :

- Sourire, joie :-)

- Colère, tristesse :-(

- Clin d'œil complice, ironie ;-)

- Admiratif :o

- Perplexe ou renfrogné :-/

- Choqué :-O

- Angoisse, peur oOoOoOoO_OoOoOoOo

- Furieux ( $\left(\begin{array}{c}\text { FE } \\ 3 E\end{array}\right.$

-Cri :- @ ou :-V

- Pleurer :'-(

- Ivresse \%*\}

Des degrés et nuances sont possibles dans l'expression figurative des affects faciaux, tels que :

- je souris :-)

•je rigole :-)) avec une modulation variable en :-))))

- je me marre avec la bouche grande ouverte de l'éclat de rire :-D

- je suis très mécontent $\{:-($ ou : $:((()$

Étant donné l'instabilité relative de ces codes, due notamment à leur caractère oral, il existe des variantes d'une période à une autre, parfois d'un pays à un autre, d'un individu à un autre, voire selon le type de forum. Ainsi le sourire de base est :-) et peut se décliner en ") en ]]] en [' _ '] ou ( : ) > ou encore en ('v') ; il existe des variantes pour le sourire chinois $\left\langle\wedge \_\wedge>\right.$ ou nordique $):-($, ou pour le sourire mêlé de sommeil <-.-> ou tordu : -7 ou encore pour le rire bruyant et grossier ) : - ) : - ) : - )

Dans les chats de conversation ou sur certains réseaux érotiques, l'icône :-P représente une langue désirante. Les actes et plaisirs sexuels sont également figurés, comme la fellation : - ) ==== 8 le cunnilingus 區 : - ) 8 監 $\mathbf{d}$ - :, l'érection :- ) -:, la masturbation :- ) $\wedge_{-:}$; le pénis prend la forme suivante [ ] ======= $\mathbf{D}$ et le phallus, selon sa dimension, est stylisé en $\mathbf{c}=$ ou en $\mathbf{C}===:$. Le corps physique ou érotique n'échappe pas aux internautes, dont on remarquera la surprésence du corps féminin - objet de représentation phantasmée de la part des hommes : seins parfaits (o)(o) suscitant l'écarquillement des yeux du voyeur $\left({ }^{*}{ }^{*}\right)$, seins avec silicone $(+)(+)$, seins avec tétons 
vers le haut $\left(^{*}\right)(*)$ ou avec grands tétons $(@)(@)$, gros seins $(0)(0)$, seins avec piercing (0)(0), etc.

Matérialiser virtuellement le sens

Ces indices matérialisent un sentiment, un désir, un goût, une réaction, un caractère, un «autoportrait» (« réel» ou feint), une intonation vocale. Au cours de certaines situations dialoguées, l'intensité de la voix est soulignée à l'aide des caractères d'imprimerie : une écriture minuscule signifie une «petite voix » ou le chuchotement, tandis que les majuscules sont réservées pour «crier» ou insister ou encore pour signaler qu'on arrive sur le forum; les caractères normaux étant destinés au mode «parler» (Bacou 2000 : 35) ; plutôt qu'un simple « NON », les orthographes « NAN » ou «NOOOON » avec une intensité plus soutenue, renforcent une négation tournant au refus catégorique; de même peut-on percevoir une nuance entre "HAHAHA " et « HIHIHI » : si les deux énoncés disent le rire, le second est d'une tonalité plus ironique ou sarcastique. L'usage de la couleur ou des onomatopées, dans les chats, véhicule des informations subjectives, par le fait d'identifier un internaute, de rendre sensible ou de renforcer une émotion: le rouge, par exemple, signifie la colère ; un " ТОСТОСТОС " marque une présence et constitue un véritable code intégré dans le geste (virtuel) d'«entrer» dans un "salon» de conversation ( room»), tout en demandant la permission d'y accéder; ou bien il traduit une volonté de s'immiscer dans un " grenier" (ou un "placard» au Portugal) [Benedito 2002: 113] où se sont "isolées " deux personnes afin de converser tranquillement entre elles ${ }^{16}$. Un langage particulier aux chats s'est institué chez les habitués, reposant sur des techniques graphiques commodes : «A+ » = « à plus tard », «SLT » = « salut » ou « byyyyyyy » ${ }^{17}$ qui exprime en anglais le mouvement de repli, éventuellement accompagné de bisous mouillés, «muaaa »ou «mu@@@@@», ou sonores, «muaccc ».

Si bien que le dialogue est rythmé, et scandé, par des expressions réduites à des initiales substantivées (acronymes), dont la plupart dérivent de l'anglais : «BBN» = "bye-bye now ", " LOL » = "laughing out loud » équivalant au français « MDR », " mort de rire »; par des onomatopées: toc toc (frapper), ZZZZzzz (ronflement); par des abréviations phonétiques : «occupé » devient « $\mathrm{OQP}$ », « $\mathrm{U}$ » résume le «you » ${ }^{18}$, avec des formes intermédiaires de suppressions, notamment de voyelles, par apocope, syncope et autre aphérèse : "jsuis ", "ordi » (ordinateur), "pq» (parce que). Cette stylisation du langage se retrouve sur le minitel rose («bz» pour «baiser »), ou sur les actuels téléphones portables conçus afin d'envoyer et de recevoir des messages numériques (SMS), et dont une publicité en 2001 interrogeait, sur le mode de l'injonction : «parlez-vous texto ? » : «G K C LA KÈCE », « POURKOI TAN DE N ? ».

Une présence de soi sensible : corps et visage

Cette écriture oralisée et abrégée ${ }^{19}$ relève de l'expressivité, c'est-à-dire de la forme sociale du lien (ludique, humoristique, ironique, sérieux, etc.), laquelle est irréductible, encore une fois, à l'expression communicante (le sens). L'expressivité met en scène le sujet par le biais de son corps signifiant -ici virtualisé et substitué sur un mode graphique- notamment la gestualité et la voix. La conversation écrite sur le multimédia se fait alors sensible.

«À travers l'écrit sourdent des formes d'échanges, des modes de socialité qui relèvent bien davantage d'une sensorialité propre au toucher qu'ils ne s'apparentent aux seuls codes abstraits des signes linguistiques. Les jargons, les détournements de caractère, l'alphabet "smiley" tirent le texte vers le geste pour instaurer le contact, créer la proximité, abolir la distance. D’abstraits les signes 
tendent à devenir figuratifs, empruntant davantage aux indices, à la trace, aux métalangages [...]. Le langage -et non plus la langue- se fait analogique, réintroduit le niveau des affects, le domaine de l'émotion, l'essence charnelle " (Sauvageot 1996 : 216).

L'éloignement des êtres favorise la réinscription d'une présence corporalisée, c'est-àdire expressive et expérientielle (Falk 1994: 62-66), grâce à des opérations symboliques : graphies, codes, affects. Ces marqueurs sont à la fois des substituts et des prolongements de l'individu-substrat, ses doubles en quelque sorte. Ils rendent possible, par-delà (et à travers) la machine, une co-présence sociale et symbolique grâce à une médiation homologique et technologique apparentée à la magie.

Ce n'est pas un hasard si c'est le visage que les smileys figurent puisqu'il est une métaphore de l'humain, l'empreinte de la subjectivité et le lieu des affects. Au travers des grimaces, des mimiques faciales et des lignes expressives, au travers de la voix qui perce, une personne ${ }^{20}$ inscrit sa présence corporelle, par le biais de la surface immatérielle de l'écran, et produit des effets. Ces codes d'écriture donnent à lire une éthique de la communication dépassant la simple parole pour focaliser sur un bienvivre-ensemble : la convivialité sur le Net. Cette éthique reste, dans la vie courante, à l'état implicite sous la forme d'un ethos. Dans les «écrits d'écran " (Souchier 1996), l'énoncé et le mode d'énonciation des affects se confondent puisque le face-à-face physique, véhiculant des informations non verbales essentielles au contrôle des interactions sociales, en est absent ${ }^{21}$.

40 À terme, la présente étude conduit à faire deux précisions conceptuelles entremêlées : sur la notion d'esthétique et sur la nature du corps.

4 La notion d'esthétique a été élargie en la soumettant, sans paradoxe, à une réduction. $\mathrm{Au}$ registre du beau, est préférée l'exploration de la matérialité du monde, de l'expressivité ordinaire et de la créativité, de l'expérience sensible engageant la raison, le corps et les affects. À la place du jugement de goût, on a préféré rendre l'esthétique à la pratique et aux relations humaines, où elle doit être saisie aussi bien. Appréhendée sous cet angle, l'esthétique ne surgit pas comme une touche supplémentaire " coloriant » les êtres et les choses, ou leurs relations. Elle est, au contraire, une forme sensible à l'œuvre au sein de l'existence, subjective ou collective, lui conférant une épaisseur et une dimension irréductibles à la rationalité et à la fonctionnalité.

Une seconde distinction porte sur le corps. Pour la vision computationnelle de la pensée, la cognition ressemble, par analogie, à un ordinateur (programmation, mémoire, fonctions). Mais pour les tenants de la conscience en tant que système dynamique, comme Timothy van Gelder (2003 : 365-369), elle est liée au déroulement temporel, elle s'avère externalisée dans les opérations sensori-cognitives du corps pensant et indexée à l'environnement. De son côté, contre la froide raison, computationnelle ou délibérative, Antonio Damasio (2001 : 12-15 et passim) montre que la capacité de raisonner est indissociable des émotions ressenties et exprimées (elles en constituent même le substratum), lesquelles reflètent les sensations du paysage corporel, lui-même en lien avec le dehors -ce tout qu'est le corps et le cerveau interagit avec l'environnement physique et social formant, ensemble, des éléments interdépendants. Ces deux conceptions anti-mentalistes rejettent la conception dualiste et hiérarchique de Descartes (et ses avatars contemporains), celle d'une pensée intérieure valorisée, atemporelle et substantielle, comme elles s'opposent à un intellect inerte et surplombant le corps. 

geste, entre l'objet-ordinateur et le sujet-utilisateur qui déploie un savoir-faire habile et non systématique, relevant du flou, de l'à-peu-près, du bricolage créatif autant que des routines. Voisinent ainsi deux ontologies : un corps somatique et un corps virtuel. Étant en résonance, comme peuvent l'être le réel et l'imaginaire, ils constituent de ce fait deux modalités du même « moi » aux contours labiles. Loin de disparaître, le corps se virtualise en redéployant ses lignes, ses limites et son mode d'être social. Ce processus de réalisation en devenir à partir du numérique recourt à une raison sensible, par quoi la visualisation substitue - partiellement- le voir et le tactile, le toucher. Ce qui émerge c'est une autre corporéité, c'est-à-dire une autre forme sociale du corps et, finalement, de l'être humain. socialement fondé où s'invente une configuration du lien : communicationnel, sensible, esthétique et politique.

\section{BIBLIOGRAPHIE}

Atifi, Assan

2003. « La variation culturelle dans les communications en ligne : analyse ethnographique des forums de discussion marocains ", Langage \& Société 104 : 57-82.

Auray, Nicolas

1999. «L'émergence du civisme dans une collectivité technique », pp. 55-71, in M.-P. Julien et J.-

P. Warnier (eds), Approches de la culture matérielle. Corps à corps avec l'objet. Paris : L'Harmattan.

Bacou, Mihaela

2000. « La parole et le lien. Communication médiatisée par ordinateur », Cahiers de littérature orale $47: 13-41$.

Benedito, Joviana

2002. Que Lingu@ Portugues@ no Chat da Internet? (Antologia). Lisbonne : Éditions Colibri.

Berger, René

1996. « Art et technologie au seuil du XXIe siècle : de l'apoptose à la métamorphose », pp. 143-164, in M. Borillo et A. Sauvageot (eds), Les cinq sens de la création. Art, Technologie, Sensorialité. Seyssel : Champ Vallon (« milieux »).

Biebuyck, Brunhilde

2000. « Du folklore au cyberlore : Paroles électroniques, avez-vous donc une âme ? », Cahiers de littérature orale 47 : 43-94.

Bourriaud, Nicolas

1998. Esthétique relationnelle. Paris : Les presses du réel.

Caune, Jean

1997. Esthétique de la communication. Paris : Presses Universitaires de France (« Que sais-je? »).

Techniques \& Culture, 42 | 2004 
Certeau, Michel (de)

1990. L'invention du quotidien 1. Arts de faire. Paris : Gallimard, 1990 (1ère édition 1980).

Classen, Constance

1997. «Fondements pour une anthropologie des sens », Revue Internationale de Sciences Sociales

(UNESCO) 153 : 437-449.

Conein, Bernard, Dodier, Nicolas \& Laurent Thévenot

1993. Des objets dans l'action : de la maison au laboratoire. Paris : EHESS (« Raisons pratiques »).

Couchot, Edmond

1996. « Des changements dans la hiérarchie du sensible. Le retour du corps ", pp. 127-131, in

M. Borillo et A. Sauvageot (eds), Les cinq sens de la création. Art, Technologie, Sensorialité. Seyssel :

Champ Vallon (« milieux »).

Damasio, Antonio

2001. L'erreur de Descartes. La raison des émotions. Paris : Odile Jacob.

Falk, Peter

1994. The Consuming Body. Londres : Sage Publications.

Forest, Fred

1998. Pour un art actuel. L'art à l'heure d'Internet. Paris : L'Harmattan (« Ouverture philosophique »).

Foucart, Stéphane

2000. « Les technologies numériques olfactives », Le Monde interactif, 5 juillet, p. 5.

Gelder, Timothy van

2003. « Dynamique et cognition », Philosophie de l'esprit : vol. II Problèmes et perspectives. Paris : Vrin

(1ère édition 1998).

Jamard, Jean-Luc

2000. « Au cœur du sujet : le corps en objets?», Techniques \& Culture 39 : 211-247.

Kant, Emmanuel

1982. Critique de la Faculté de Juger. Paris : Vrin (1ère édition 1790).

Kisseleva, Olga

1998. Cyberart. Un essai sur l'art du dialogue. Paris : L'Harmattan (« Ouverture philosophique »).

Labov, William

1993. « Les insultes rituelles », pp. 391-456, Le parler ordinaire. La langue dans les ghettos noirs des Etats-Unis. Paris : Éditions Minuit.

Le Breton, David

1999. L'adieu au corps. Paris : Métailié.

Lelong, Benoît

2002. «Savoir-faire technique et lien social. L'apprentissage d'internet comme incorporation et autonomisation », pp. 267-292, in C. Chauviré et A. Ogien (eds), La régularité. Habitude, disposition et savoir-faire dans l'explication de l'action. Paris : EHESS.

Leroi-Gourhan, André

1965. Le Geste et la Parole, tome I. Paris : Albin Michel.

Lévy, Pierre

1997. Cyberculture. Rapport au Conseil de l'Europe. Paris : Odile Jacob.

1998. Qu'est-ce que le virtuel ?. Paris : La Découverte/Poche. 
Lire [magazine]

2000. « Comment la technologie modifie l'écriture », n²84, pp. 50-63.

Longavesne, Jean-Paul

1996. « Machines à peindre et informatique picturale », pp. 96-103, in M. Borillo et A. Sauvageot

(eds), Les cinq sens de la création. Art, Technologie, Sensorialité. Seyssel : Champ Vallon (« milieux »).

Majastre, Jean-Olivier

1999. "Transfusion de sens », pp. 163-174, in Approche anthropologique de la représentation. Entre

corps et signe. Paris : L'Harmattan.

Marcoccia, Michel

2000. «Les smileys : une représentation iconique des émotions dans la communication médiatisée par ordinateur ", pp. 249-263, in C. Plantin, M. Doury, et V. Traverso (eds), Les émotions dans les interactions. Lyon : Presses Universitaires de Lyon.

Mercury, Jean-Yves

2000. L'expressivité chez Merleau-Ponty, du corps à la peinture. Paris : L'Harmattan.

Merleau-Ponty, Maurice

1945. Phénoménologie de la perception. Paris : Gallimard.

Millet, Agnès

1998. « La ville : un espace socio-sémiotique », pp. 25-42, in V. Lucci, Vincent, A. Millet, J. Billiez, J.-P. Sautot, N. Tixier (eds), Des écrits dans la ville. Sociolinguistique d'écrits urbains : l'exemple de Grenoble. Paris : L'Harmattan.

Moles, Abraham

1990. Art et ordinateur. Paris : Blusson.

Ninio, Jacques

1989. L'empreinte des sens : la raison perceptive. Paris : Odile Jacob.

Norman, Donald A.

1993. «Les artefacts cognitifs", pp. 15-34, in B. Conein, N. Dodier et L. Thévenot (eds), Les objets dans l'action : de la maison au laboratoire. Raisons pratiques. Paris : EHESS.

Otman, Gérard

1999. Les mots de la cyberculture. Paris : Belin.

Pierozak, Isabelle

2000. « Approche sociolinguistique des pratiques discursives en français sur internet : "ge fé dais fotes si je veux" ", Revue Française de Linguistique Appliquée V (I), 2000 : 89-104.

2003. « Le "français tchaté" : un objet à géométrie variable ? ", Langage \& Société 104 : 123-144.

Pina Cabral, João

1998. « Redes informáticas - espaços, tempos, hegemonias », Análise Social XXXIII, 148-4

(Lisbonne) : 861-870.

Rheingold, Howard

1995. Les Communautés virtuelles. Paris : Addison Wesley France (1ère édition 1993).

Rigaut, Philippe

$A u$-delà du virtuel. Exploration sociologique de la cyberculture. Paris : L'Harmattan (« cultures sensibles »).

Rouet, Jean-François

2004. Cent fenêtres sur internet : Rapport final. www.msh.univ-poitiers.fr/laco/ 
Sansot, Pierre

1986. Les formes sensibles de la vie sociale. Paris : Presses Universitaires de France.

1995. La France sensible. Paris : Petite Bibliothèque Payot (1ère édition 1985).

Sauvageot, Anne

1996. «Art, Technologie et recomposition du sensible », pp. 211-218, in Borillo, Mario, et

A. Sauvageot (eds), Les cinq sens de la création. Art, Technologie, Sensorialité. Seyssel : Champ Vallon

(« milieux »).

2003. L'épreuve des sens. De l'action sociale à la réalité virtuelle, Paris: Presses Universitaires de

France (« Sociologie d'aujourd'hui »).

Simmel, George

1981. «Essai sur la sociologie des sens », pp. 223-238, in Sociologie et Epistémologie. Paris : Presses

Universitaires de France.

Simondon, Georges

1989. Du mode d'existence des objets techniques. Paris : Aubier (1ère édition 1958).

Souchier, Emmanuel

1996. « L'écrit d'écran », Communication et langages 107.

Warnier, Jean-Pierre

1999. Construire la culture matérielle. L'homme qui pensait avec ses doigts. Paris : Presses

Universitaires de France (« Sciences sociales et sociétés »).

\section{NOTES}

1. Ce texte concerne une recherche en cours. La présente étude, bien que restituant des matériaux dérivés de plusieurs chats français et portugais récents (2000-2002), de nature ludique et/ou érotique, est surtout d'orientation conceptuelle. Les idées développées ici seront éprouvées dans une analyse ultérieure, plus ethnographique. 2. On parle d'art informatique, de création artistique numérique, de cyberart, voire d'informatique picturale. Sur le cyberart, consulter Forest (1998), Kisseleva (1998) et Sauvageot (2003).

3. Dans la Critique de la Faculté de Juger, Kant (1982 : § 20 à 22) définit le sensus communis comme le principe subjectif qui fonde universellement par sentiment -et non pas par concept comme dans la connaissance- un jugement de goût particulier; en sorte que, par la recherche d'un tel accord interne, une belle chose n'est pas belle pour moi, ni même en soi, mais doit " plaire universellement sans concept » (§ 16). Remarquons que Kant, à côté des jugements esthétiques purs qui portent uniquement sur la forme de la beauté de l'objet ou de sa représentation, distingue les jugements esthétiques empiriques, qu'il écarte du beau, tels que ceux relevant de l'agréable ou du désagréable (plaisir, peine...), et les nomme des « jugements esthétiques matériels » limités aux sens (§ 14). C'est justement cette esthétique matérielle, ce « reste barbare » de la beauté que nous mettons au centre de la réflexion.

4. Parmi les travaux portant sur l'expressivité centrée sur le corps et le monde, ceux de Merleau-Ponty (1945) sont essentiels; voir aussi Mercury (2000).

5. Sur la cyberculture et ses potentialités créatrices en matière d'art, qui modifie son mode d'être et sa nature, se reporter à Lévy (1997), en particulier le chapitre IX : « L'art et la cyberculture ».

6. Voir Warnier (1999) et la discussion engagée par Jamard (2002). 
7. La notion de virtualisation forgée par Lévy (1998: 9-10) ne s'oppose pas au " réel », pas plus qu'elle ne fraye avec le «faux »; elle est moins une réalité « en puissance », par opposition à ce qui est « en acte », qu'une puissance de devenir portée par une indétermination positive, un mode d'être fécond et un processus global et incessant de transformation, lequel remonte du réel vers le virtuel afin de « réaliser » l'hominisation. C'est dire que s'il y a un « donné » ou un « monde », ce n'est qu'en tant que processus. Le concept de virtualisation peut être rapproché de celui de «technogenèse » proposé par Berger (1996: 148) et qui « instaure un pouvoir de réalité ».

8. Le terme de « couplage » entre l'homme et la machine est à entendre dans le sens que Simondon lui a donné : un certain état atteint à partir d'une « synthèse » des deux entités qui ne forment plus qu'une seule fonction complexe.

9. Il faudrait ici nuancer et relativiser. Dans un article consacré aux « Réseaux informatiques -espaces, temps, hégémonies ", l'ethnologue portugais Pina Cabral (1998) rappelle des évidences anthropologiques face à des enthousiasmes « communautaristes » ou " modernistes » un peu naïs, sinon infantiles (comme chez René Berger). L'auteur émet un doute quant à l'uniformisation du « monde » avec le « nouvel ordre global »; un doute face à l'idée d'une communauté virtuelle pensée en dehors de représentations collectives partagées et comme « incarnées » et reposant sur des interactions interindividuelles ; un doute à propos d'un outil d'où toute stratégie de pouvoir et de contrôle serait absente ou à l'égard de la notion de " culture globale » venant contredire la diversité des sociétés et leur éventuelle coexistence. Pina Cabral observe que l'on confond souvent ce qu'il appelle les « cultures organiques », fondées sur un cadre de vie habituel et des dispositions familières, et les "supracultures » recourant, occasionnellement, à d'autres conceptions, à d'autres usages, techniques et codes sociaux, tels que ceux du multimédia (la « cyberculture »). Sur deux exemples de relativisation culturelle de la communication entre internautes dans des forums (avec des salutations, des thèmes, des surnoms, des expressions affectives ou des injures spécifiques) ainsi que de l'usage ou du mésusage des règles afférentes, voir Atifi (2003) pour le Maroc et Benedito (2002) pour le Portugal où la « langue internaute », outre un style sui generis, est un mélange de portugais académique, d'argots, de régionalismes, de brésilien (lusitanisé) avec l'influence des feuilletons (telenovelas), de castillan, de français (déformé) et de dialectes africains caractéristiques des influences et du multiculturalisme postcolonial du pays.

10. Si, dans l'existence courante, les sens sont continuellement sollicités : toucher pour « voir » si l'eau du bain est assez chaude, goûter pour vérifier l'état de la cuisson ou la teneur en sel d'un plat, sentir un aliment pour décider s'il faut le garder ou le jeter ou, simplement, pour découvrir sa saveur, il reste que la multiplication des techniques dans la vie quotidienne, introduisant des commandes à distance, des manettes, des boutons, des claviers ou, dans un registre plus sophistiqué, sollicitant la voix ou la présence physique (pour allumer une lumière ou fermer une porte), si elles désensibilisent le rapport aux choses et aux êtres, ne le décorporéisent pas, car l'action motrice s'installe au milieu de ces objets et de ces ambiances qui, en retour, lui assurent des prises.

11. En 1999 des chercheurs de l'Institut Weizman, en Israël, ont découvert une méthode qui devrait rendre possible, à travers un équipement électronique, la transmission des odeurs « en direct » par les moyens de communication. Cette communication olfactive, procédant par « capture » et «traduction » technologiques des saveurs, permettra aux 
utilisateurs de faire l'expérience d'odeurs par le biais de l'ordinateur, de la télévision, du téléphone ou de l'écran du cinéma. Sur les technologies numériques olfactives, voir aussi Foucart (2000 : 5). Par ailleurs, il est aujourd'hui possible d'être prévenu vocalement -et pas seulement de voir/lire- de l'arrivée d'un nouveau message sur son e-mail, avec le «Speaking Email Deluxe ».

12. Un besoin de voir et de toucher, sinon même d'écrire à l'encre et de raturer dont témoignent la plupart des écrivains qui travaillent sur écran ; $c f$. le dossier du magazine Lire (2000) : «Comment la technologie modifie l'écriture ».

13. Il faudrait pouvoir repérer les différents usages de l'ordinateur, notamment les « détournements » ludiques et civiques opérés par les hackers, les « pirates » de l'informatique (Auray 1999); comme il faudrait pouvoir distinguer ses usages selon les moments et les milieux sociaux ainsi que les types d'adaptation avec des manières et une habileté acquises par un apprentissage formel ou tacite, tâtonnant et personnalisé, qui relève davantage de la pratique des règles que des règles de la pratique : ce que Le Long (2002) appelle les « prises » et le "régime de familiarité » indexé au corps sensoriel spatialisé et au monde des objets. Voir plus largement l'ouvrage de Conein, Dodier et Thévenot (1993). Par ailleurs, les dépanneurs en informatique rapportent des situations décalées et cocasses auprès d'utilisateurs débutants, conseillés par téléphone : une confusion de vocabulaire, comme entre le sens technique de «fenêtre » et la fenêtre du domicile que le client va «fermer » sous injonction ou bien un mauvais emploi des outils, comme l'utilisation au pied de la "souris ", ou des fonctions, comme de vouloir introduire un $\mathrm{CD}$ dans le lecteur de disquettes, etc. Sur les usages et mésusages de l'ordinateur, voir Rouet (2004) la seule étude disponible à ce jour. 14. Une telle hypothèse doit sans doute être restreinte à certains groupes sociaux ou professionnels (amis, famille, collègues, etc.) ; elle appelle en tous les cas une vérification. Nous envisageons de la reprendre dans une étude centrée sur un espace de travail spécifique. Nous nous intéresserons, en particulier, aux nombreuses histoires racontées, aux blagues narrées (souvent sexistes et sexuelles, rendues possibles par l'interconnaissance comme par la distance physique ou celle de l'humour et de l'écrit), aux rumeurs rapportées et autres paroles et graphismes figuratifs (dessins, etc.) circulants et anonymes transitant par le e-mail. Cette production balance entre la créativité « spontanée », le ludique et le lien sensible. Voir sur ces aspects Bacou (2000) et Biebuyck (2000).

15. Pour la classification, les fonctions et les usages circonstanciés des smileys, se reporter à Marcoccia (2000: 254-260). Bacou (2000:31) signale que dans certains forums de discussion, avec le temps se sont instaurés des procédés et un jargon parmi les « initiés » face aux nouveaux venus baptisés «newbies » (bleus).

16. Remarquons que room, grenier, placard, chat réfèrent au foyer et s'identifient à de la convivialité, à de l'appartenance affective et à une politesse conversationnelle d'où tous les racismes sont, formellement, exclus.

17. Pierozak (2003: 133) observe que « les allongements graphiques, qui consistent à répéter (au-delà du doublement) le même graphème, concernent surtout les pseudos des habitués et s'observent essentiellement à l'ouverture et à la clôture des échanges [...]. Ces procédés font partie des rituels de salutation puisqu'ils permettent d'évaluer et d'afficher indirectement un statut vis-à-vis de la communauté : plus le tchateur est salué, plus il est fait usage d'allongements... et plus il est "important" ».

18. Sur l'écriture internet, voir Otman (1999). Il faudrait rapprocher ou même comparer ce graphisme pragmatique et virtuel de l'écriture murale des graffiteurs et 
graphistes qui combinent en une même unité « icono-graphique » (Millet 1998: 49) l'écrit cursif à déchiffrer et l'image visuelle, d'autant que la technologie la plus récente permet d'associer ou, plutôt, de combiner de façon inventive et judicieuse, texte et image.

19. Sur cette question, voir Pierozak (2000).

20. Le mot persona en latin désigne, on le sait, la " personne » et le masque personnifié dans le théâtre ; par le biais du masque perce le son de la voix (per-sona), marquant l'importance de cet organe. La voix, comme le visage, est porteuse de l'identité. 21. Outre les smileys, d'autres indices relevant du contenu signifiant de la communication peuvent fonctionner comme des marqueurs sociaux. Derrière l'apparent anonymat du « pseudo » et de la théâtralisation des propos, l'identité supposée- des locuteurs surgit à la faveur d'un décodage social. C'est ainsi que procède cette utilisatrice d'un forum de conversation (Le Monde, 11/8/2000, p. 18) : «J'effectue un premier tri en fonction du pseudo [...], Goldorak ou Marteau Piqueur, très peu pour moi. Ensuite, je consulte la fiche des préférences. Si le mec cherche un physique féminin bien précis, je n'insiste pas ; il n'a aucun intérêt. Ensuite, on se rend compte rapidement si le monsieur cherche de l'« instant sex" ou encore s'il est un peu «niais". Une entrée en matière du style "Bonjour, j'ai envie de communiquer. Toi aussi ?" donne tout sauf envie de poursuivre ". Rigaut (2001 : chap. 3) remarque que la « maîtrise de l'orthographe, de la syntaxe (dont les critères d'appréciation sont problématiques dans la mesure où l'exigence de rapidité impose l'usage d'abréviations et une cadence olympique propice aux erreurs de frappe), le type de langage qu'il emploie, ses lapsus, oublis et contradictions en tous genres, peuvent livrer quantité d'informations "sociologiques" sur le chatteur ». Le choix du " pseudo », sur les réseaux sexuels ou conversationnels, n'est pas toujours de pure invention : il s'appuie parfois sur différentes facettes de l'identité « réelle » (goûts, humour, connaissance des règles du jeu), qu'il emprunte à l'univers érotique ou au monde de la BD et de la SF, quand ce n'est pas tout l'être qui se joue de soi, grâce à la distance communicante et au masque du désir/plaisir. On le voit, le « qui » parle va de pair avec le « comment» on choisit de parler (Bacou 2000 : 34).

\section{RÉSUMÉS}

Cet article aborde la créativité ordinaire sur ordinateur et les usages de graphismes codifiés, les smileys. Ceux-ci expriment les affects des internautes au cours des interactions médiatisées par l'ordinateur, marquant une téléprésence sensible et un triple lien : ludique, éthique et esthétique. L'expressivité du visage et la sexualité sont les deux principales sphères qui émergent de cette interactivité. Contrairement à l'idée d'une disparition postmoderne du corps, avec le numérique, la corporéité se trouve transfigurée tout en demeurant une dimension symbolique centrale dans les échanges scripturaires et graphiques sur les chats, ces espaces virtuels de conversation, ainsi que dans les usages cognitifs de l'ordinateur.

Usual aesthetic and chats : computer, corporality and codified expression of affects. This article deals with ordinary creativity through computer and with the smileys (codified graphics), 
which express the net surfers' emotions (when they interact through computers), pointing out a telepresence and a triple link : playful, ethical and aesthetic. The face expressiveness and sexual signs are the most represented on the chats. The numerical system, in opposition to the postmodern idea of a desappearance of the body, transfigures the corporality which keeps a central symbolic dimension in the exchanges on the chats and in the cognitive uses of computers.

Estética ordinaria y chats. Computadora, corporeidad y expresión codificada de las emociones. El artículo enfoca la creatividad corriente mediante las computadoras -los ordenadores- y las prácticas de los grafismos codificados, los smileys. Esto últimos expresan las emociones de los internautas durante las interacciones mediatizadas por la computadora. Evidencian una telepresencia sensible y una relación de naturaleza triple : lúdica, ética y estética. La expresividad de la cara y la sexualidad son las dos esferas principales que sobresalen en el marco de parecida interactividad. Al revés de la idea de una desaparición posmoderna del cuerpo, con lo numérico, la corporeidad se trasfigura sin dejar de ser una dimensión simbólica central en los intercambios epistolares y gráficos por chats, estos espacios virtuales de conversación. Eso vale también para los usos cognitivos de la computadora.

INDEX

Mots-clés : esthétique, ordinateur, chats, corporéité, multimédia, smileys

\section{AUTEUR}

\section{ANIBAL FRIAS}

Chercheur affilié au Laboratoire d'anthropologie sociale, CNRS. 